\title{
Research on Initial Filter Criteria of IP Multimedia Subsystem
}

\author{
Yafang WANG \\ School of Information Science and Engineering \\ Hebei University of Science and Technology \\ Shijiazhuang, China \\ e-mail: wangyafang@hebust.edu.cn
}

Leilei KANG

School of Information Science and Engineering

Hebei University of Science and Technology

Shijiazhuang, China

e-mail: lei-736@163.com

\author{
Xiaozhe ZHENG \\ School of Information Science and Engineering \\ Hebei University of Science and Technology \\ Shijiazhuang, China \\ e-mail: zhengxiaozhe12@163.com \\ Bingyang CHENG \\ School of Information Science and Engineering \\ Hebei University of Science and Technology \\ Shijiazhuang, China \\ e-mail: 13703313380@163.com
}

\section{IFC OVERVIEW}

\begin{abstract}
IMS offers a unified service trigger mechanism, whose core is triggering the service to the corresponding SIP application server which based on initial filter criteria. Then the exclusive SIP application servers provide various valueadded multimedia services for the users. This paper researches how to create the exclusive service data which is Initial Filter Criteria, when a user receives an IMS subscription which contains specific value-added services. Then the SIP request initiated can be implemented routing to the appropriate application server accurately.
\end{abstract}

\section{Keywords-IMS; iFC; Service Triggering; XML}

\section{INTRODUCTION}

IMS (IP Multimedia subsystem) is a system put forward in support IP multimedia service by the 3rd Generation Partnership Project in Release five which provide the IP Multimedia service unified system structure and infrastructure for mobile communication construction, especially the service control with multifunction and access network independence based on SIP (session initiation protocol). It makes IMS get the approval of all the communication standard agency, and will gradually become the core of the next generation network.In addition, it is gradually applied to the sevices which provided by the operators. The provider of service is done by the home network in the IMS network. That means it is the home network S-CSCF to complete service triggering control, and then forwarded to the corresponding application server for the processing of sevice logic when the user roaming. The mode of S-CSCF control enables the control layer and service layer of IMS is completely separated, therefore IMS service triggering mode becomes a spot in research. S-CSCF controls service triggering of information according to the filter criteria configured in the HSS, and the service triggering data is downloaded to the S-CSCF during the registration phase or no registered users launch or accept sessions.So this paper will analyze initial filter criteria deeply.

\section{A. Definition and Background}

IFC: Initial Filter Criteria. It is Used to describe when routing to a specific application server about the SIP message received, and it is the main content of the existing IMS service triggering data, furthermore it is also the core of IMS service triggering mechanism. S-CSCF is responsible for analyzing iFC in IMS network, and then routes the SIP request to the specified application server. IMS itself do not provide value-added services. On the contrary, it is a system based on SIP, and it can be realizing on advanced IP services and applications in PS network. IMS provide the necessary methods for application and service. Operators need a user profile assigned to the user when he gets a IMS subscription. The configuration includes the user's private identity and public identity, as well as public identity associated the iFC [1]. The service configuration is stored in HSS. And the information is downloaded to S-CSCF when the user registered to network. S-CSCF does not need to care about what the users signed service but only does iFC matching detection when dealing with customers request. If the information that the user provided is in line with the triggering condition, S-CSCF pass the message to the specified application server what make AS can complete the service of user signed [2]. IMS could achieve the separation of control layer and the application layer through iFC.

\section{B. Scene of Triggering AS}

\section{1) Triggering in registration process}

According to the Register message, the SIP request is triggering to the application servers when the user registration. This is the user AS signing service, and it is SCSCF in IMS network. S-CSCF download user configuration data to HSS through the SAR request when registering. So the user can trigger to the AS for service processing when making call and other services. AS may lead to business failure because of no business data if 
calling to trigger AS rather than registering to trigger AS before. The scene of triggering AS is shown in Fig. 1.

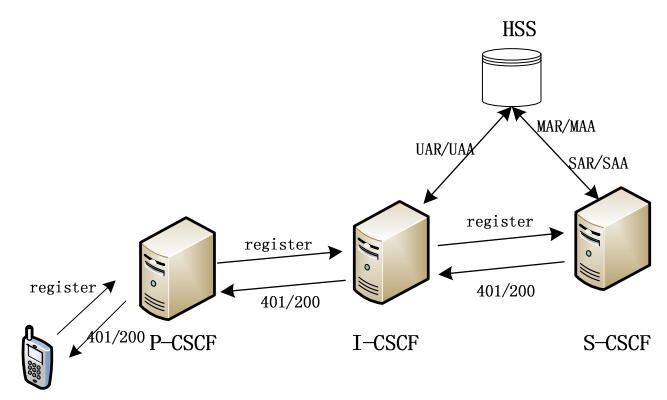

Figure 1. Scene of triggering AS in registration process

\section{2) Triggering in calling process}

The calling and called users can both trigger AS in order to contract services, such as customized ringing tone, call transfer and all services provided by third party server. Normally the AS refers to IM-SSF in application layer of IMS, OSA SCS and SIP AS. S-CSCF saved the user configuration data download from HSS during the process of registering. Then it can trigger AS directly to carry on services when calling. The scene of triggering AS is shown in Fig. 2.

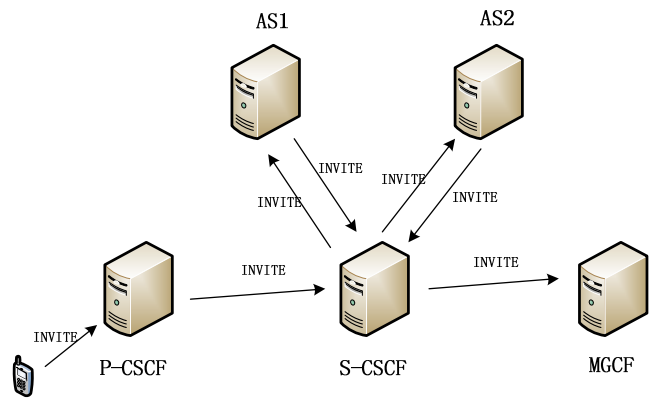

Figure 2. Scene of triggering AS in calling process

\section{IFC FRAMEWORK}

\section{A. Service Profile Structure and Composition}

In the IMS service system, each IMS subscriber has its own Service Profile. The Service Profile includes the data related service triggering, such as Initial Filter Criteria and Shared iFC Set. These service attributes associated with particular users stored in HSS, and it downloads to S-CSCF through Cx interface when the user registers. It is S-CSCF to evaluate whether the SIP request fulfill the filter criteria.

The UML model of a user data is shown in Fig. 3. In the IMS system, it is used SAR/SAA message download to SCSCF from HSS through the Cx interface (Section 2.2 Triggering AS in registration process Scene), IMS Subscription contains a Private User Identity, one or more Service Profile [3].

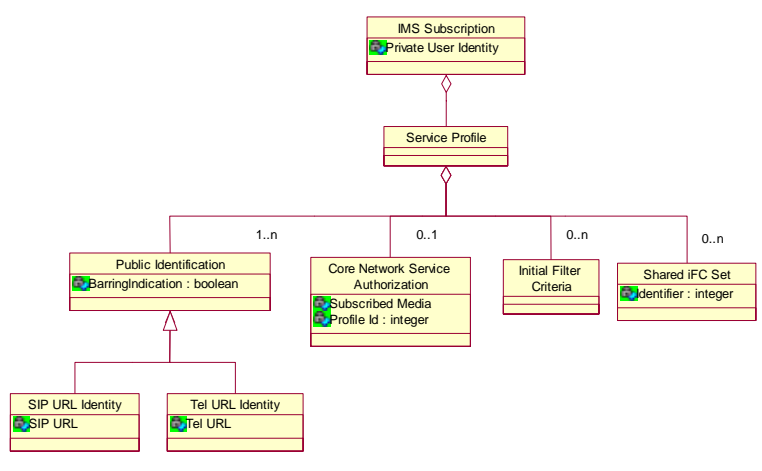

Figure 3. UML model of user data

A Service Profile includes: Public User Identity (one or more), Core Network Service Authorization Information (zero or one), Initial Filter Criteria information (zero or more) and Shared iFC Set Information (zero or more). And in one Service Profile, the Core Network Service Authorization Information, Initial Filter Criteria Information and the Shared iFC Set Information can be used by all the users with Public User Identity. Public User Identity can be SIP URL format or Tel URL format. In system of IMS, all communication needs Public User Identity. The Barring Indication belongs to the boolean type, if it was seted, the S-CSCF will ban the users to use all the services in the IMS domain except the registration and re-registration service. Core Network Service Authorization consists of subscribed media strategy information and service level. If there is no Core Network Service Authorization Information, it's no need to subscrib the Filter Criteria which concerned with media in the SCSCF. A Initial Filter Criteria includes zero or numbers of iFC triggering rules. Based on the iFC, S-CSCF matches user's SIP requests and then routes them to the corresponding application server; the Shared Initial Filter Criteria Set is a collection of Initial Filter Criteria of local management, which is a iFC subscription collection that can be shared by more than one Service Profile. Core Network Service Authorization, Initial Filter Criteria and Shared iFC Set are bounded with Public User Identity. The difference between Shared iFC set and iFC is that: HSS transfer a ID to S-CSCF, and the ID represents a group of iFC in Shared iFC Set. This group of iFC in HSS and S-CSCF are predefined and consistent, thus can save interface flows of HSS and S-CSCF.

\section{B. IFC Structure and Composition}

As the most important part stored user information in the network, iFC decides what service provided to each user.iFC includes a number of user information, and the user information assists S-CSCF to decide whether to get a special AS to provide services. The composition of iFC is shown in Fig. 4. 


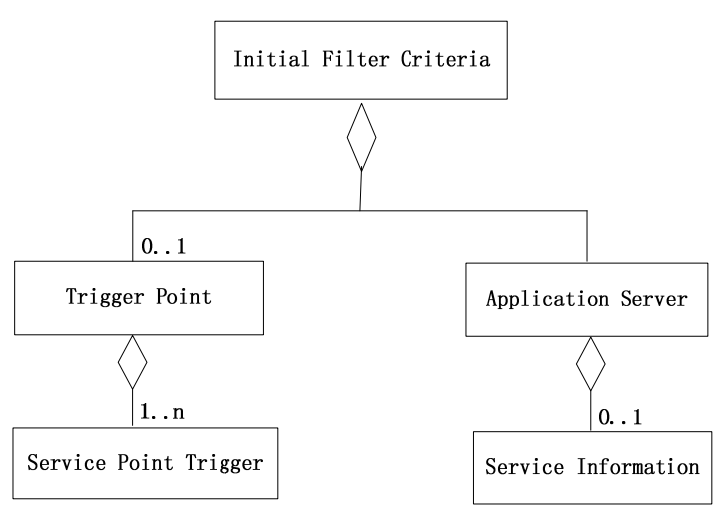

Figure 4. iFC framework

An initial Filter Criteria includes zero or one trigger point and an application server. Each initial Filter Criteria of service configuration has a unique priority in S-CSCF. When assigning a plurality of initial Filter Criteria, S-CSCF evaluates the iFCs according to the priority. Value is greater, and the priority is lower. Trigger point is used to decide whether or not to contact the application server, and it contains one or more service point triggers. Service point trigger is a set of logical judgment. It shows that triggering the AS unconditionally if no trigger point. Application server defines which the application server address to contact when trigger point is matched. Application server includes zero or one Service Information. During the registration period, Service Information allows SIP requst to be sent to AS through S-CSCF transparently when initial Filter Criteria condition is satisfied. Application server can also include what to do with the related session default processing information when connecting with AS failure.

\section{Trigger Point Structure and Composition}

Trigger point is used to check whether the message is matched to a particular application server, so as to decide whether or not to contact the AS. Each trigger point contains one or more service point trigger instance. And each service point trigger instance is a "and" or "or" description of boolean expression [4]. iFC defines five kinds of triggers in IMS network. The structure of triggers is shown in Fig. 5.

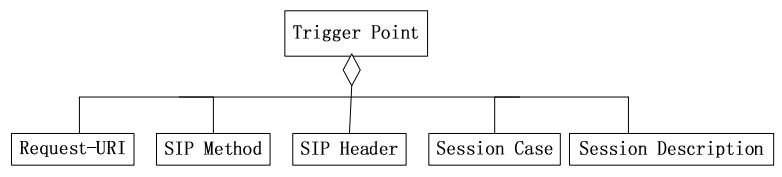

Figure 5. Structure of service point trigger

Request URI: Identifying the resource which the request directing to.iFC can trigger according to vary URI requests.

SIP Method: Representing the type of user request, such as INVITE and Message. IFC can trigger according to vary request types.

SIP Header: It contains header or header content of the user request message, such as the From Header. A service point trigger can decide whether there are any SIP Headers, or includes any SIP header's content. iFC can trigger according to different header or header content.

Session Case: Representing the session state, such as calling registration process and called unregistered process. It may have three kinds of value: Originating, Terminating or Terminating_Unregistered, and they indicate whether the filter should be used by S-CSCF to process end-user's services of Originating, Terminating or erminating_Unregistered. iFC can triggering according to vary session process.

Session Description: Representing any SDP content in SIP Method. iFC can triggering according to vary different media information [5].

Each trigger points can include five kinds of service point trigger which can be Request URI, SIP Method, SIP Header, Session Case, and Session Description. It represents that whether to trigger AS judging from the five aspects. Then take some examples to analysis the five kinds of trigger.

1) RequestURI Trigger

The trigger which RequestURI is “sip:test@ims.com” triggering AS is as follows.

$<$ SPT $>$

$<$ ConditionNegated $>0</$ ConditionNegated $>$

$<$ Group $>0</$ Group $>$

$<$ RequestURI $>$ sip:test@ims.com $<$ RequestURI $>$

$</$ SPT $>$

SPT represents Service Point Trigger.The tag of " $<$ SPT $></$ SPT $>$ " contains a trigger,and a trigger point which tagged with “ $<$ TriggerPoint $><$ TriggerPoint $>$ " can contain multiple triggers;

ConditionNegated represents the conditions which trigger meets, 0 shows group condition does not invert, and 1 shows group condition invert;

Group is used to divide the triggers into groups, 0 shows the trigger belongs to the zeroth group. And each group may contain multiple triggers.

2) SIPMethod Trigger

The trigger which service request message type is "INVITE" triggering AS is as follows.

$<$ SPT $>$

$<$ ConditionNegated $>0<$ ConditionNegated $>$

$<$ Group $>0</$ Group $>$

$<$ Method $>$ INVITE $</$ Method $>$

$</$ SPT $>$

3 ) SIPHeader Trigger

The trigger which FromHeader does not contain "ims.com" in service request message triggering AS is as follows.

$<$ SPT $>$

$<$ ConditionNegated $>1<$ ConditionNegated $>$

$<$ Group $>1<$ Group $>$

$<$ SIPHeader $>$

$<$ Header $>$ from $</$ Header $>$

$<$ Content $>$ ims.com $</$ Content $>$

$<$ /SIPHeader $>$

$</ \mathrm{SPT}>$

Here ConditionNegated is 1 , then group condition invert, shows to do SPT with invert operation. 


\section{4) SessionCase Trigger}

The trigger which represented Terminating_Unregistered process is as follows.

$<$ SPT $>$

$<$ ConditionNegated $>0</$ ConditionNegated $>$

$<$ Group $>1</$ Group $>$

$<$ SessionCase $>2</$ SessionCase $>$

$$
</ \mathrm{SPT}>
$$

"SessionCase" descrips different cases of the session, the possible value of 0 indicates Originating, and 1 indicates Terminating while 2 indicates Terminating_Unregistered.

\section{5 ) SessionDescription Trigger}

The trigger which the line of $m$ contains "ABC" in session SDP media information is as follows.

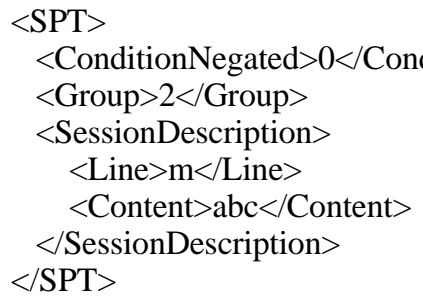

\section{IFC XML CODING INSTANCE}

In the process of service control, initial Filter Criteria decides the services provided to each user, and it assists SCSCF to decide when to introduce a special application server to provide services. The configuration information which contains initial filter criteria using XML document to describe. Now, this paper researches the XML configuration examples of initial filter criteria with detail description. The first task is to clear the iFC configuration principle about the iFC configuration data. First of all possible service or service group should be defined, then create the special user service data, and finally trigger the incoming initial request to the AS. Before the planning of iFC, need to understand the network deployed of IMS service type, then make a plan of the service trigger sequence, what's more, understand the service characteristics is as important.

\section{A. Trigger Point Instance}

Trigger point is composited with one more triggers. Assume that conversation scene is Terminating_Unregistered, then trigger with the filter criteria is for receiving INVITE message and SessionCase is 2, so the trigger point of XML configuration of triggering AS is follows:

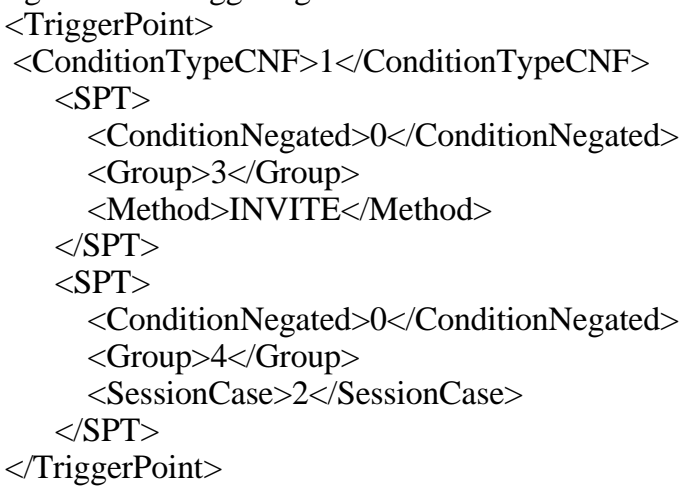

ConditionTypeCNF shows the group relationship of each trigger, and it used to indicate that expression format of SPT set. The representation type of service point trigger instance is Boolean. If it is true, the TP Boolean expression is a Conjuctive Normal Form (CNF); if it is false, the TP Boolean expression is a Disjunctive Normal Form (DNF). 0 is the internal Group 'and', representes 'or' between groups; 1 is the internal Group 'or', representes 'and' between groups. In this example, there are two triggers belonging to the 3rd group and the 4th group. ConditionTypeCNF is 1 showing that two groups must be satisfied at the same time.

\section{B. IFC Instance}

IFC is composed of trigger points and application server. If it is Terminating_Unregistered, and triggering to SIP AS (as1@as.ims.com) when calling, and triggering the application server fails, then make conversation continues. The iFC configuration is as follows:

$<$ InitialFilterCriteria $>$

$<$ ProfilePartIndicator $>$ REGISTERED $<$ /ProfilePartIndi cator $>$

$<$ Priority $>0</$ Priority $>$

$<$ TriggerPoint $>$

$<$ TriggerPoint $>$

$<$ ApplicationServer $>$

<ServerName>sip:as1@as.ims.com</ServerName> ultHandling $>$

$<$ DefaultHandling $>$ SESSION_CONTINUED $</$ Defa

$<$ ServiceInfo $>$ GTAS $</$ ServiceInfo $>$

$</$ ApplicationServer $>$

$</$ InitialFilterCriteria $>$

Using < InitialFilterCriteria> </InitialFilterCriteria $>$ to mark an initial filter criteria. ProfilePartIndicator used to mark the service registered or unregistered, which belongs to the type of the enumeration. And its possible values is REGISTERED, UNREGISTERED and PARTIND_DEFAULT. In this case, it is REGISTERED, and triggering after registered. So it indicates that the iFC belongs to part of registered user profile [6]. Priority representes the iFC priority, and in this case, the value is 0 which representing the highest priority, so S-CSCF should deals with the SIP message first. What omitted in $<$ TriggerPoint $><$ /TriggerPoint $>$ is the contents of the XML code of trigger point instance in the 4.1 section. ServerName representes the AS address triggering to. DefaultHandling representes the default process when triggering fails. It is the type of enumeration, and the possible values is SESSION_CONTINUED, SESSION_TERMINATED and SESSION_ROUTED. In this case, the value is SESSION_ CONTINUED what represents leting the session to continue when the application server can't arrived. That means if there is a low priority iFC exist, then continue. ServiceInfo stands for Service Information, and it allows to download some information to S-CSCF from HSS. When the trigger point condition is satisfied, the information can be transparently transferred to the application server. 


\section{TEST AND VERIFICATION}

According to the service providing structure of IMS and the research about initial Filter Criteria above, basing on the OpenIMSCore test bed, developed online billing server and the calling hidden server which based on SIP. Configuring the shared iFC so that it can be applied to the other users subscribing the same service.The OpenIC soft terminal, PCSCF, S-CSCF, HSS and two SIP servers deployed online billing and calling hidden server connected together. IMS calling subscriber whose sip address is +8613800000001@ims.com need to trigger the online billing server AS1 (sip:ocg1.ims.com) to online billing first when calling, then trigger AS2 (sip:espace1.ims.com) for calling hidden, and then route the calling to the CS domain of the called subscriber. It will end the call if triggering failure. The test topology is shown in Fig. 6.

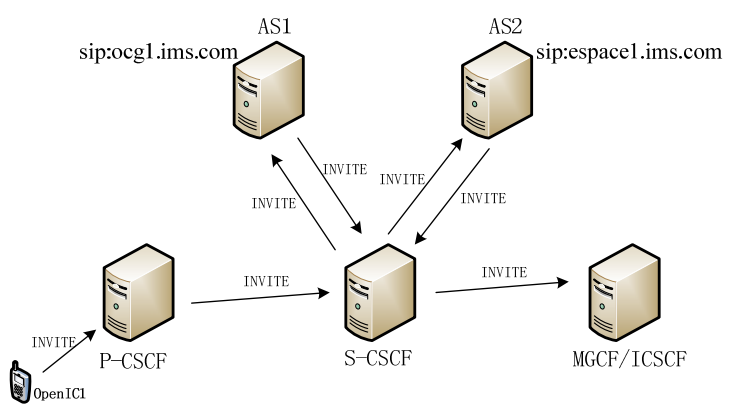

Figure 6. Test topology

And the configuration information of S-CSCF is as follows.

1) Add the iFC triggering to the online billing gateway in the Shared iFC Set:

ADD SIFCINF: SIFCTPLID=99, IFCNAME="Online charging",

PARTIND=PARTIND_DEFAULT,

SERVER="sip:orig@ocg1.ims.com",

DEFHND=SESSION_TERMINATED,

TRIGPT $=$ " $<$ TriggerPoint $>$

$<$ ConditionTypeCNF $>0</$ ConditionTypeCNF $>$ $<$ SPT $>$

$<$ ConditionNegated $>0</$ ConditionNegated $>$ $<$ Group $>0<$ /Group $>$

$<$ Method $>$ Invite $<$ /Method $>$ $</$ SPT $>$

$</$ TriggerPoint $>$ "

2) Add the iFC triggering to the calling hidden AS in the Shared iFC Set:

ADD SIFCINF: SIFCTPLID=99, IFCNAME="CLIR", PRIORITY=1,
SERVER="sip:espace@espace1.ims.com", DEFHND=SESSION_TERMINATED, TRIGPT=" $<$ TriggerPoint $>$

$<$ ConditionTypeCNF $>0</$ ConditionTypeCNF $>$ $<$ SPT $>$

$<$ ConditionNegated $>0</$ ConditionNegated $>$ $<$ Group $>0</$ Group $>$

$<$ Method $>$ Invite $<$ /Method $>$ $</$ SPT $>$

$<$ TriggerPoint $>"$

Finally, turn on the switch which supporting shared iFC set of the S-CSCF, and execute the command in CSCF as follows:

MOD SCSCF: SID=0, SHAREDiFC=SUPPORTED

The test results: The data transmitting between the two terminal shows normal, and the online billing AS and the calling hidden AS work fine. Initial Filter Criteria can be matched the SIP message correctly, and routes it to the appropriate application server accuracy. Hence all the test results accord with expected.

\section{CONCLUSION}

IMS has won the approval of numerous standard organizations. Many domestic and international communicational manufacturers have also devoted to the research of IMS. And it began to be applied into businesses. So the scale of its development will be enlarged in the future. This paper researches on initial filter criteria of IMS from some aspects. And its researching result laid foundation for the further study of the service triggering.

\section{ACKNOWLEDGMENT}

In the process of researching iFC, I appreciate the great help and support from the other teachers in Communications Department and my student. Particularly my student Zheng Xiaozhe invested a lot of energy reviewing literature and analysing experiment in the subject.

\section{REFERENCES}

[1] 3GPP TS 22.228 v.7.10.0. Service requirements for the Internet Protocol (IP) multimedia core network subsystem, R7, 2007.

[2] 3GPP TS 23.002 v7.4.0. Network architecture, 2007.

[3] 3GPP TS 29.228 v7.7.0. Annex B: User profile UML model, 2007.

[4] L. Hu, L. Cao, J. Chen, "IMS Technology Principle and Application," Beijing: Electronics Industry Press, 2006, pp. 126-168.

[5] 3GPP TS 24.229 v7.10.0. IP Multimedia Call Control based on Session Intiation Protocol (SIP) and Session Description Protocol (SDP), 2007.

[6] M. Poikselka, G. Mayer Eds., Y. Wang, W. Dong, S. Zhou, "IMS: IP Multimedia Concepts and Services,” Beijing: Mechanical Industry Press, 2007, pp. 382-435. 